\title{
Normal Hepatocyte Transplantation Delays the Emergence of Chemically Induced Preneoplastic Nodules in Rat Liver
}

\author{
Maria Paola Serra, Silvia Doratiotto, Fabio Marongiu, and Ezio Laconi \\ Department of Biomedical Sciences, Unit of Experimental Medicine, University of Cagliari, Cagliari, Italy
}

\begin{abstract}
Cancer often arises in a background of chronic tissue damage. It is also increasingly appreciated that such an injured tissue microenvironment might foster the selective emergence of altered cells, leading to neoplasia. Accordingly, reversal of chronic tissue damage could represent a potential strategy to counteract neoplastic disease. In these studies, we aim to investigate whether transplantation of normal cells in the context of an injured, neoplastic-prone microenvironment might impact on the evolution of the carcinogenic process. A rat model of chemically induced hepatocarcinogenesis was used. Animals were given a single dose of diethylnitrosamine (DENA), followed by two injections of retrorsine (RS), a pyrrolizidine alkaloid that imposes a persistent block on hepatocyte cell cycle. At the end of this protocol, rats were either given no further treatment or injected, via the portal circulation, with 4 million normal hepatocytes isolated from a syngenic donor. After 3 months, rats given DENA+RS alone displayed numerous discrete nodular lesions (up to 30 per liver), ranging 1 to $3 \mathrm{~mm}$ in size. On the other hand, in animals receiving DENA+RS and transplantation, donor hepatocytes were able to repopulate over $50 \%$ of the host liver, as expected. Most importantly, both the number and the size of hepatocyte nodules were greatly reduced in these animals (percent nodular area was $1.8 \pm 0.3$, down from a control value of $8.5 \pm 2.8$ ). The above data indicate that strategies aimed at reestablishing a normal tissue microenvironment might be relevant to the management of neoplastic disease.
\end{abstract}

Key words: Cell transplantation; Tumor microenvironment; Cell competition; Liver repopulation; Liver carcinogenesis

\section{INTRODUCTION}

The pathway towards cancer in many organs, including the liver, is often inextricably linked to chronic disease processes that hamper the functional capacity of the tissue, and also its regenerative potential $(7,9)$. For example, liver cirrhosis, the single most important risk factor for hepatocellular carcinoma, is associated with impaired liver regeneration both in humans $(20,21)$ and in experimental animals $(5,11)$. Thus, it is reasonable to hypothesize that such a growth-impaired background could provide a favorable competitive landscape for the selective emergence of rare cells with altered/preneoplastic phenotype $(4,8,18,26)$. Consistent with this interpretation, studies from our laboratory have indicated that hepatocytes isolated from chemically induced liver nodules can rapidly proliferate and progress to cancer following transplantation into a growth-constrained liver microenvironment, while they are unable to grow to any significant extent when injected into the liver of a normal, syngenic recipient (16).

An important corollary of the hypothesis above is that strategies aimed at normalizing a chronically injured and/or growth-constrained tissue environment might be of value in the management of neoplastic disease (18). The present study was designed to test this possibility. Normal hepatocytes were transplanted in the setting of an irreversibly injured, neoplastic-prone microenvironment; donor-derived cells were able to extensively repopulate the host liver and this effect was associated with a significant decrease in the incidence of chemically induced hepatocyte nodules.

\section{MATERIALS AND METHODS}

\section{Animals and Treatments}

Liver carcinogenesis was induced using a sequential exposure to diethylnitrosamine (DENA) and retrorsine

Received July 26, 2010; final acceptance April 25, 2011. Online prepub date: September 22, 2011.

Address correspondence to Dr. Ezio Laconi, M.D., Ph.D., Dipartimento di Scienze Biomediche, Unità di Medicina Sperimentale, Via Porcell 4, III piano, Università di Cagliari, 09124, Cagliari, Italy. Tel: +39 070675 8342; Fax: +39 070 662574; E-mail: elaconi@unica.it 
(RS), a pyrrolizidine alkaloid that imposes a persistent block on normal hepatocyte cell cycle $(14,25)$. We predicted that a population of DENA-initiated hepatocytes might be able to withstand the mitotic block imposed by RS and selectively proliferate vis-à-vis the surrounding parenchymal cells; such prediction was also based on data available for other alkaloids related to RS (10).
Male Fischer 344 rats, 4 weeks of age, were injected with DENA (160 mg/kg, IP), followed by two doses of RS (30 mg/kg each, IP), given at 2 and 4 weeks after DENA administration. Two weeks after the inducing protocol, animals were divided into two groups of eight rats each: group 1 received no further treatment, while group 2 was transplanted with $4 \times 10^{6}$ hepatocytes
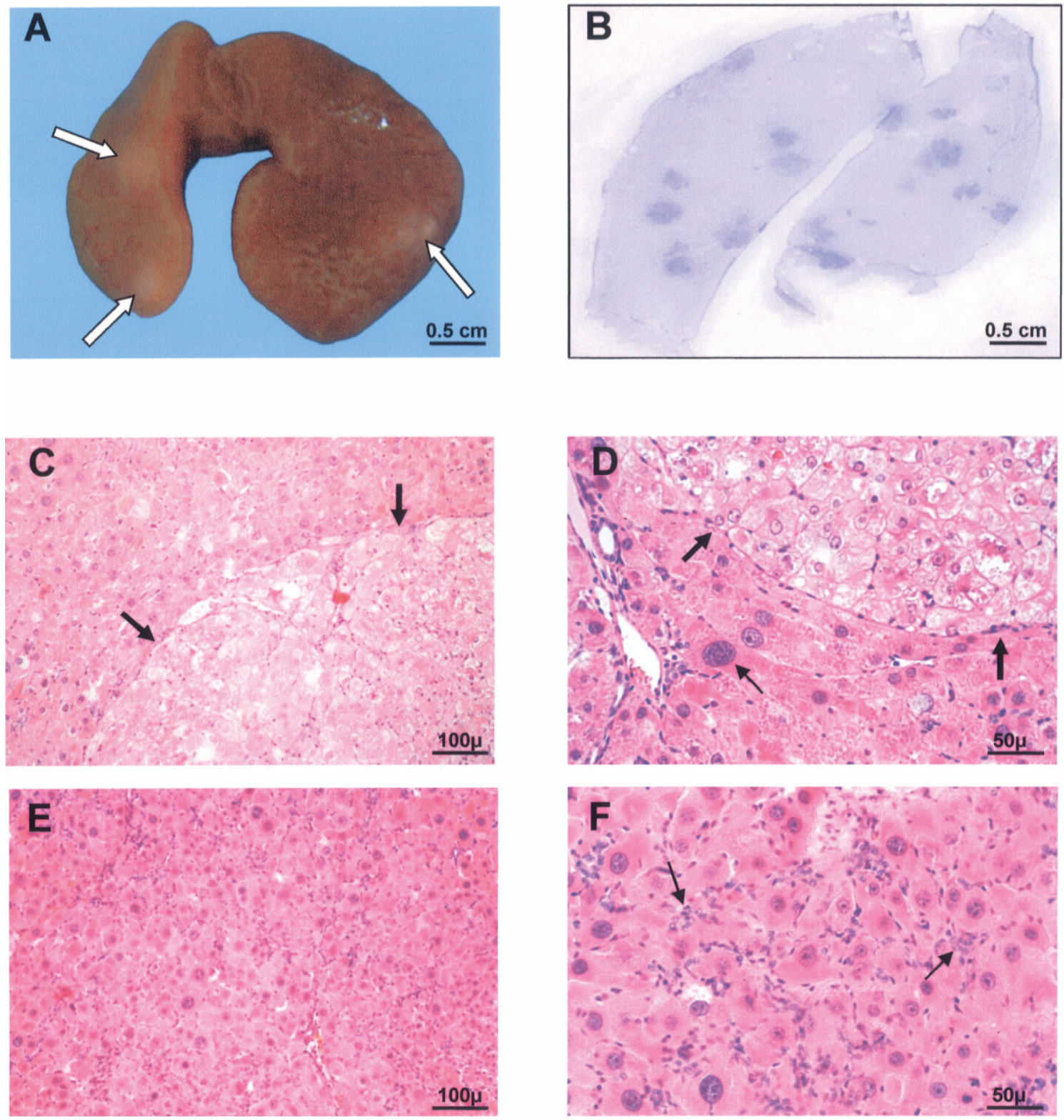

Figure 1. Liver samples of rats exposed to diethylnitrosamine plus retrorsine ((DENA+RS) and killed 4 months later (see text for details). (A) At least three white-grayish nodules (arrows) in the caudate lobe. (B) A whole cryostat section of a liver samples from the same group processed for immunohistochemical detection of glutathione-S-transferase (GST) 7-7 (see text for details); GST 77-espressing nodular lesions are stained in dark blue. $(\mathrm{C}-\mathrm{F})$ The histological appearance of H\&E-stained liver sections is shown; (C) and (D) show a liver nodule (thick arrows), sharply demarcated from surrounding enlarged hepatocytes (megalocytes, thin arrow in D). (E, F) The typical alterations induced by RS on rat liver can be observed, with extensive megalocytosis and moderate to intense proliferation of ductular epithelial cells (F, thin arrows). 

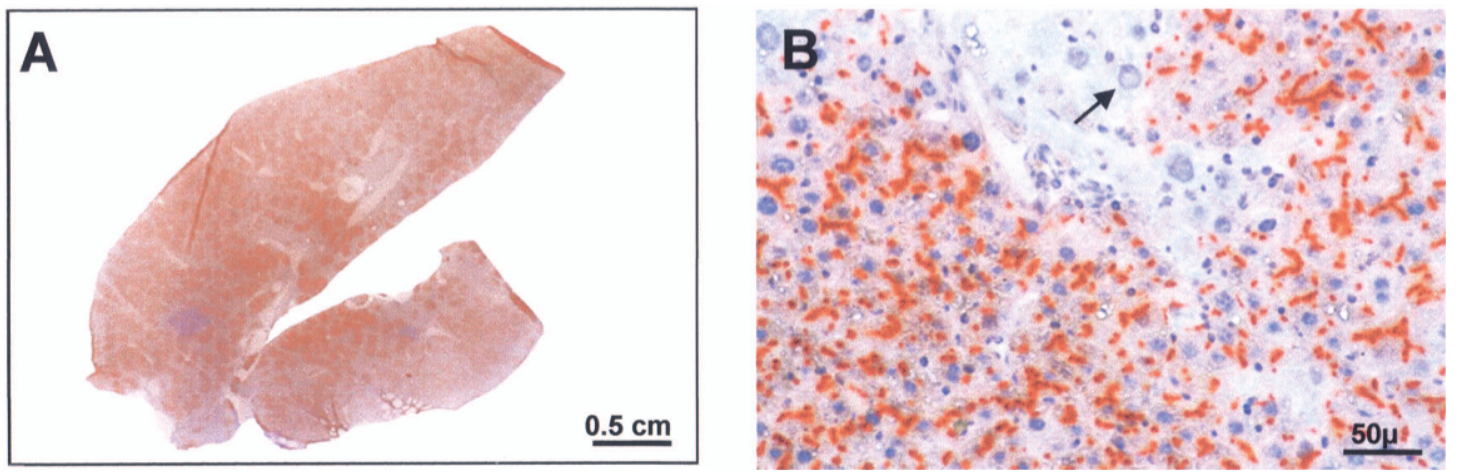

Figure 2. Liver samples of rats exposed to DENA+RS followed by hepatocyte transplantation and killed 4 months later (see text for details). (A) A whole cryostat section of a liver samples processed for dual histochemical-immunohistochemical detection of F344-dipeptidyl-peptidase Type IV (DPP-IV) activity (orange-rust) and GST 7-7 expression (dark blue) (see text for details). Note the extensive repopulation of the host liver by DPP-IV-positive, donor-derived hepatocytes. (B) A microphotograph of a transplanted liver is shown: only residual endogenous megalocytes were present (thin arrow).

isolated from a normal syngenic donor. All animals were killed 4 months after DENA administration. All experiments were approved by the University of Cagliari Ethical Committee for Animal Experimentation; all animals received humane care in accordance with NIH Guidelines for the care and use of animals.

\section{Hepatocyte Isolation and Transplantation}

Hepatocytes for transplantation were isolated according to a two-step collagenase perfusion technique $(2,22)$. Cell viability, determined by trypan blue exclusion at the end of the isolation procedure, was $>90 \%$. Hepatocytes were suspended in PBS $\left(1 \times 10^{7} / \mathrm{ml}\right)$ and were injected via a mesenteric vein.

Animals were anesthetized and a small incision (about $1 \mathrm{~cm}$ ) was performed in the upper abdominal wall; hepatocytes were then delivered through a branch of the mesenteric veins, using a syringe with a 26-gauge needle. The fate of donor-derived cells in the recipient liver was followed using the F344-dipeptidyl-peptidase type IV (DPP-IV)-deficient model for cell transplantation (24). Donor hepatocytes were isolated from animals expressing the marker enzyme (DPP-IV positive), while DPP-IV-deficient rats were used as recipients. Since the Fischer 344 rat is a syngenic strain, no immunosuppression was required for successful cell transplantation.

\section{Histochemical and Immunohistochemical Methods}

After sacrifice, livers were removed and examined for the presence of hepatic nodules or any other evident lesions. Samples were taken from each lobe and were either frozen for cryostat sections or fixed in buffered formalin for standard histological analysis and immunohistochemistry.

The incidence of preneoplastic lesions was evaluated using immunohistochemical detection of the marker enzyme glutathione-S-transferase (GST) 7-7, according to previously published methods (16). The extent of liver repopulation in transplanted animals was monitored in cryostat sections stained for DPP-IV expression, using a simple histochemical technique (16).

\section{Statistical Analysis}

Results are presented as mean \pm SE. Two-tailed Student's $t$-test was used to evaluate results, with a lowest level of significance of $p<0.05$.

\section{RESULTS}

\section{The Induction of Hepatic Nodules by Sequential Exposure to DENA+RS}

As predicted, sequential exposure to DENA and RS resulted in the occurrence of numerous hepatocyte nodules, both on the liver surface (up to 30/liver, mean 18 \pm 5 ) (Fig. 1A) and on sectioning (Fig. 1B). Nodular lesions were white-grayish in color and resembled those induced by other classical protocols of chemical hepatocarcinogenesis in the rat, both on macroscopic appearance and upon histological analysis (Fig. 1C, D). They also expressed the GST 7-7 marker enzyme (Fig. 1B), further supporting their putative preneoplastic nature.

The surrounding liver was mainly occupied by enlarged (megalocytic) hepatocytes, with rare clusters of very small hepatocytes that have been referred to as regenerative nodules (19); mild to intense proliferation of oval cells was also observed (Fig. 1E, F).

\section{Liver Repopulation by Transplanted Cells in DENA+RS-Treated Liver}

We have repeatedly shown that preexposure to RS sets the stage for extensive repopulation of the host liver by transplanted normal hepatocytes (18). However, no 

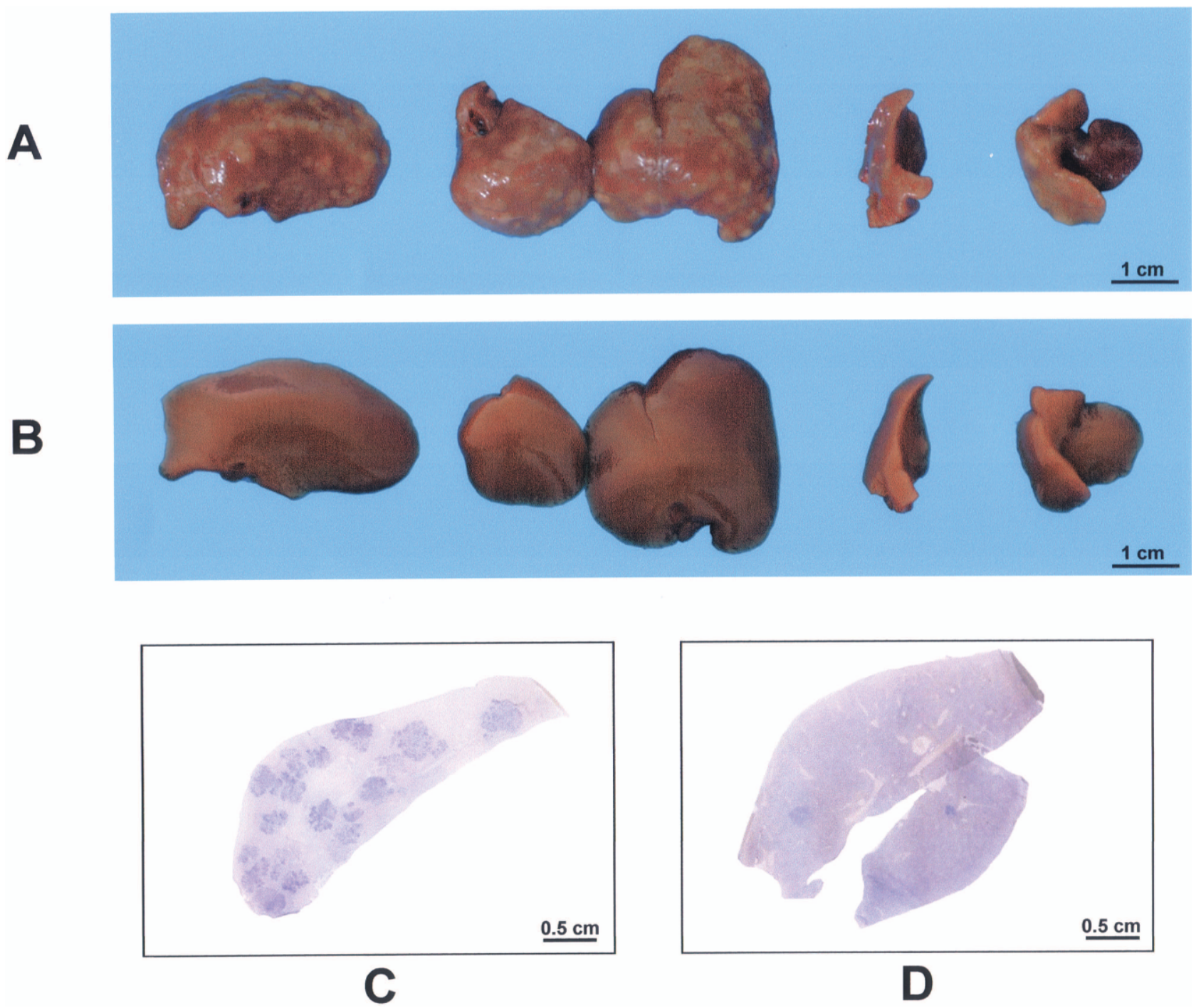

Figure 3. Liver samples of rats exposed to DENA+RS (A, C) or DENA+RS+Tx (transplant) (B, D) and killed 4 months later (see text for details). (A) DENA+RS: each liver lobe shows numerous white-grayish nodules, similar to Figure 1A; however, no such lesions are present in liver lobes in (B), taken from a rat exposed to DENA+RS+Tx. (C, D) Whole cryostat sections of the same livers processed for immunohistochemical detection of GST 7-7 (see text for details). Note the presence of several GST 7-7-positive lesions (dark blue) in the liver in (C) (DENA+RS), while few such lesions are present in (D) (DENA+RS+Tx).

studies had been published so far on the effect of a combined treatment with DENA and RS on liver repopulation.

Percent of host liver replacement by donor-derived cells was therefore evaluated in these experiments using a computer-assisted image analyzer (17). High levels of repopulation were observed in all transplanted animals, with an average of $53 \pm 7 \%$ of total liver replacement by DPP-IV-expressing hepatocytes (Fig. 2A, B). The distribution of transplanted cells clusters was generally homogeneous throughout the recipient lobes, although some areas displayed lower levels of repopulation. Combined histochemical-immunohistochemical analysis for
DPP-IV and GST 7-7 revealed that no overlapping was present between the two markers (i.e., none of the donor-derived hepatocytes clusters expressed the GST 7-7 enzyme), as previously observed (15).

The Effect of Hepatocyte Transplantation on the Incidence of Nodules in the Liver of DENA+RSTreated Rats

We next aimed to determine whether transplantation of normal hepatocytes and the associated extensive liver repopulation had any influence on the growth of nodules induced by sequential exposure to DENA and RS. Results are reported in Figure 3 and 4. The number of 

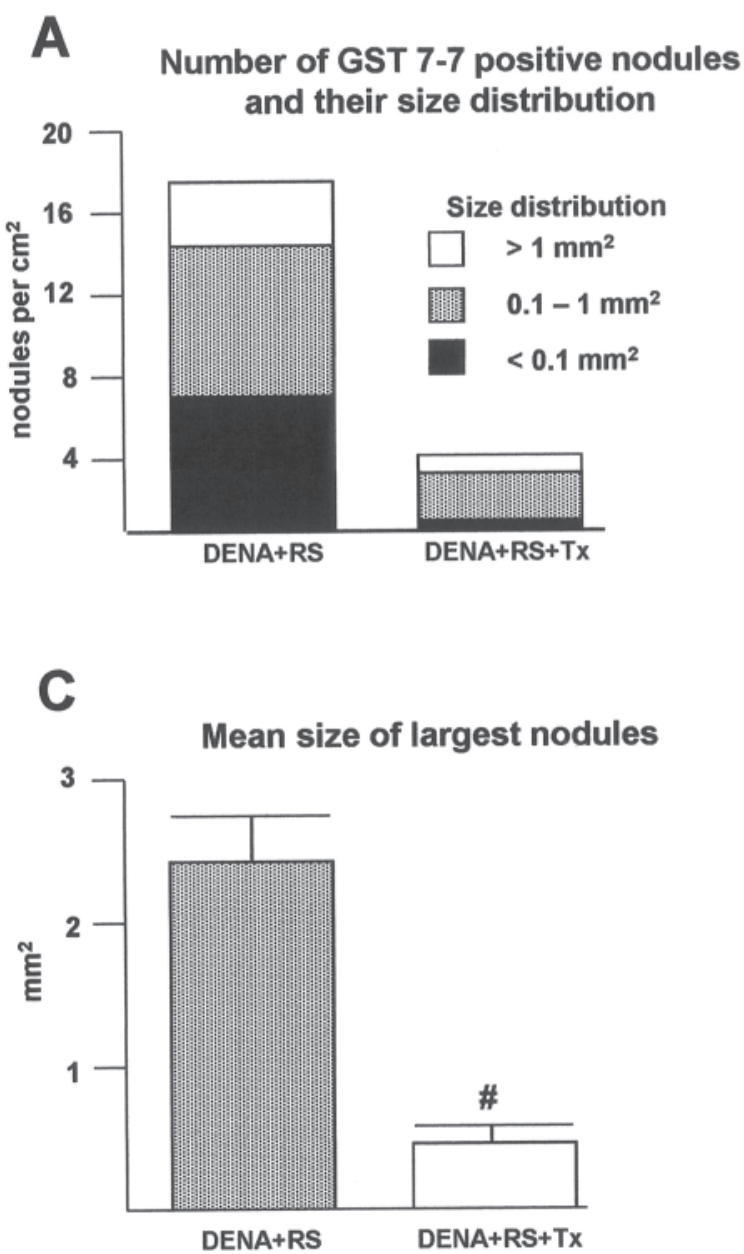

B

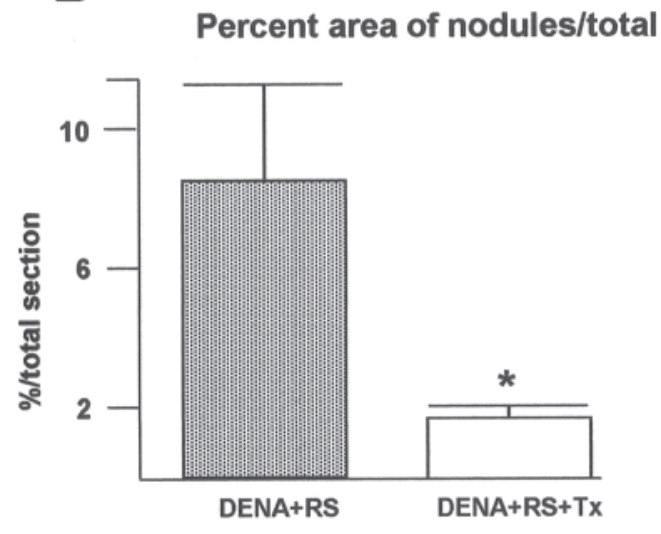

D

Percent area of repopulated liver

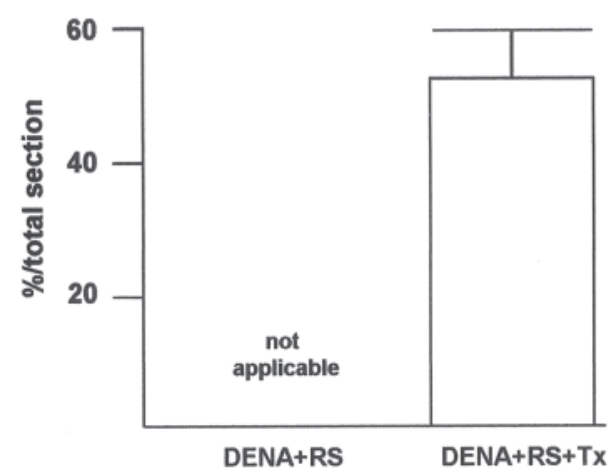

Figure 4. The incidence of GST 7-7-positive nodules was evaluated in both DENA+RS and DENA+RS+Tx groups at 4 months after starting the experiment. (A) The number and size distribution of GST 7-7 expressing lesions is compared. (B) The percent area occupied by nodular lesions in each group. (C) The three largest GST 7-7-positive nodules from each animal were considered, and their mean size was compared in the two groups. (D) The extent of liver repopulation by transplanted cells in rats exposed to DENA+RS+Tx. Data are mean \pm SE. Significantly different from control: $* p<0.05$; $\# p<0.001$ (two-tailed $t$-test).
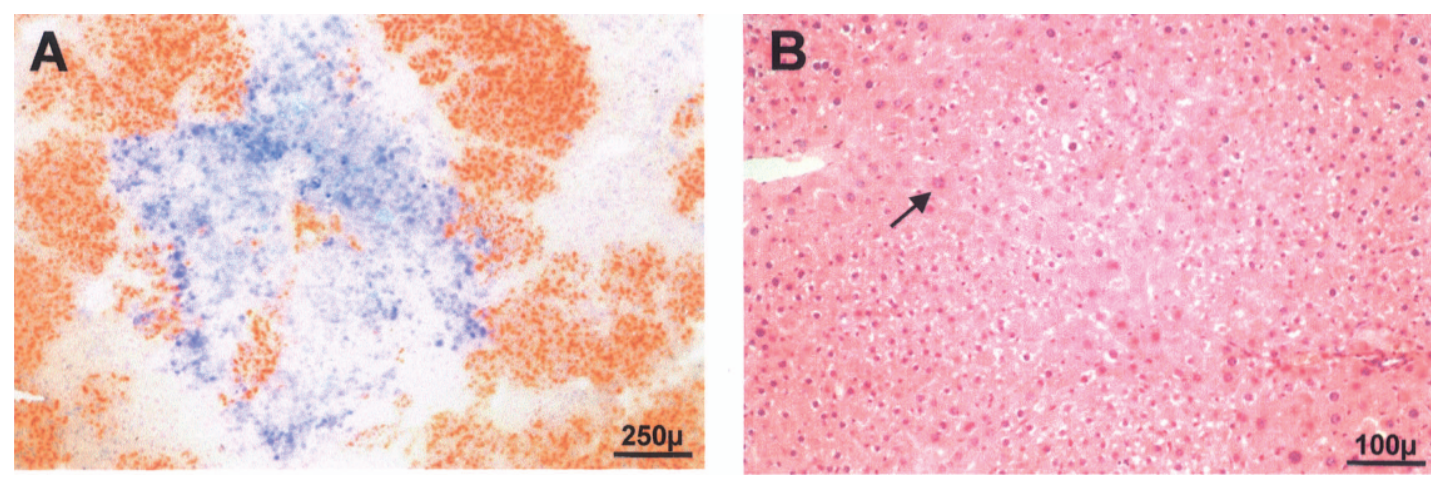

Figure 5. (A) Combined histochemical-immunohistochemical staining for DPP-IV (orange-rust) and GST 7-7 (dark blue) on cryostat liver sections from a rat exposed to DENA+RS+Tx. Note that clusters of donor-derived cells appear to be present around and/ or in the context of GST 7-7-positive nodular lesions. The surrounding host liver is unstained. No overlapping was ever observed between DPP-IV and GST 7-7 reactivity. (B) The effect of hepatocyte transplantation on liver histology is shown: only residual RS-induced megalocytes were present (thin arrow), while proliferation of bile ductular cells was greatly reduced. 
GST 7-7-positive focal lesions was greatly reduced in rats receiving DENA $+\mathrm{RS}$ and transplantation compared to the control group given DENA+RS only ( $3 \pm 2$ vs. 19 \pm 8 lesions $/ \mathrm{cm}^{2}$ ) (Fig. 4A; see also Fig. $3 \mathrm{~A}$ and $\mathrm{C}$ vs. $3 \mathrm{~B}$ and $\mathrm{D}$ for comparison). This was also reflected in the relative area occupied by nodular lesions, below $2 \%$ in the group receiving DENA+RS and transplanted cells $(1.8 \pm 0.3 \%)$, down from a control value of $8.5 \pm 2.8 \%$. This represents a net decrease of almost $80 \%$ between group 1 and group 2 (Fig. 4B). Furthermore, the mean size of the three largest GST 7-7-positive nodules found in each animal of the two groups was reduced by a factor of about 5 in transplanted versus nontransplanted rats (Fig. 4C).

Interestingly, DPP-IV-positive hepatocyte clusters were occasionally seen inside nodular, GST 7-7-expressing lesions (Fig. 5A), suggesting the possibility that donor-derived normal hepatocytes might integrate into hepatic nodules and disrupt their continuity.

The histology of transplanted and repopulated livers was also remarkably different compared to the nontransplanted group. Only residual chords of megalocytes were seen in between clusters of donor-derived cells. The latter displayed normal size, normal histology, and were perfectly integrated into the host liver, with no evident demarcation from resident hepatocytes (Figs. 2B, 5B). Proliferation of oval cells was also greatly reduced in livers receiving hepatocyte transplantation (Fig. 5B).

Parameters related to liver size and liver DNA content in both transplanted and nontransplanted groups were also measured. No significant differences were seen in final body weight and liver weight. Interestingly, a slight increase in liver DNA content was observed in the group receiving transplantation $(6.42 \pm 0.12$ vs. 5.64 $\pm 0.34 \mathrm{mg}$ DNA/liver/per $100 \mathrm{~g}$ b.w., $p<0.05)$.

\section{DISCUSSION}

The unequivocal information that emerges from these studies is that transplantation of normal hepatocytes in the setting of a neoplastic-prone liver microenvironment is able to exert a significant impact on the development of nodular preneoplastic lesions. Injection of $4 \times 10^{6}$ hepatocytes, isolated from a normal syngenic donor, into the liver of a recipient animal preexposed to a carcinogenic protocol, resulted in a sharp delay and/or reduction in the incidence of hepatocyte nodules. This effect was accompanied by an extensive repopulation of the host liver by transplanted cells, resulting in a profound modification of the neoplastic-prone tissue landscape.

It is being increasingly appreciated that carcinogenesis is not a cell-autonomous process; rather, the phenotypic behavior of preneoplastic and even neoplastic cell populations appears to be critically dependent on specific cues provided by the surrounding microenvironment
$(3,6,13)$. This emerging paradigm is of particular relevance to liver cancer, given the fact that hepatocellular carcinoma most often arises in a background of chronic injury, with the ensuing inflammation, repair, fibrosis, and cirrhosis (1). Such a reactive microenvironment is likely to contribute critical factors for the selective emergence of cells with altered phenotype on the pathway towards cancer. Within this context, transplantation studies conducted by our group have firmly established the essential role of the microenvironment in determining the growth behavior of altered/nodular hepatocytes, as referred to in the Introduction (18).

The present studies add to those findings and provide a "proof of principle" that an irreversibly altered tissue microenvironment does in fact represent a driving force during early stages of carcinogenesis. Most importantly, our data indicate that the role of the microenvironment is potentially amenable to modulation. In fact, transplantation of normal cells was able to reverse, at least in part, tissue alterations induced by the carcinogenic protocol and this effect was associated with a sizeable reduction in the incidence of preneoplastic nodules.

No definitive mechanism(s) can be proposed at this time to explain this phenomenon. One attractive possibility is that the extensive structural remodeling of the host liver resulting from normal hepatocyte transplantation may help renormalize the neoplastic-prone microenvironment induced by DENA and RS, as suggested by stem cell transplantation studies $(12,23)$. This in turn would translate into a decreased selective pressure for the emergence of altered cells. Alternately, or in combination, transplanted normal cells may compete with resident altered hepatocytes for the availability of critical growth factors that are essential for both cell types, thereby limiting the growth of the latter. In a recent review we discussed the intriguing analogies existing between liver repopulation and carcinogenesis, and suggested that in fact these two processes may share common pathogenetic mechanisms (10). Within this conceptual framework, normal cells could possibly be used to competitively counteract the selective growth of altered cells during early stages of carcinogenesis, as described in the present studies.

In summary, we provide evidence to suggest that strategies aimed at normalizing the microenvironment of a chronically and/or irreversibly injured liver tissue prone to cancer may help reduce the risk of neoplastic disease.

ACKNOWLEDGMENTS: Grant support: AIRC (Italian Association for Cancer Research) Grant No. IG4927 and IG10604. The authors declare no conflict of interest.

\section{REFERENCES}

1. Ascha, M. S.; Hanouneh, I. A.; Lopez, R.; Tamimi, T. A.; Feldstein, A. F.; Zein, N. N. The incidence and risk factors 
of hepatocellular carcinoma in patients with nonalcoholic steatohepatitis. Hepatology 51:1972-1978; 2010.

2. Berry, M. N.; Friend, D. S. High-yield preparation of isolated rat liver parenchymal cells: A biochemical and fine structural study. J. Cell Biol. 43:506-520; 1969.

3. Bissell, M. J.; Radisky, D. Putting tumours in context. Nat. Rev. Cancer 1:46-54; 2001.

4. Chao, D. L.; Eck, J. T.; Brash, D. E.; Maley, C. C.; Luebeck, E. G. Preneoplastic lesion growth driven by the death of adjacent normal stem cells. Proc. Natl. Acad. Sci. USA 105:15034-15039; 2008.

5. Chijiiwa, K.; Nakano, K.; Kameoka, N.; Nagai, E.; Tanaka, M. Proliferating cell nuclear antigen, plasma fibronectin, and liver regeneration rate after seventy percent hepatectomy in normal and cirrhotic rats. Surgery 116:544-549; 1994

6. Davalos, A. R.; Coppe, J. P.; Campisi, J.; Desprez, P. Y. Senescent cells as a source of inflammatory factors for tumor progression. Cancer Metastasis Rev. 29:273-283; 2010.

7. De Marzo, A. M.; Nakai, Y.; Nelson, W. G. Inflammation, atrophy, and prostate carcinogenesis. Urol. Oncol. 25: 398-400; 2007.

8. El-Serag, H. B.; Rudolph, K. L. Hepatocellular carcinoma: Epidemiology and molecular carcinogenesis. Gastroenterology 132:2557-2576; 2007.

9. Fox, J. G.; Wang, T. C. Inflammation, atrophy, and gastric cancer. J. Clin. Invest. 117:60-69; 2007.

10. Hayes, M. A.; Roberts, E.; Farber, E. Initiation and selection of resistant hepatocyte nodules in rats given the pyrrolizidine alkaloids lasiocarpine and senecionine. Cancer Res. 45:3726-3374; 1985.

11. Kaido, T.; Yoshikawa, A.; Seto, S.; Yamaoka, S.; Sato, M.; Ishii, T.; Imamura, M. Portal branch ligation with a continuous hepatocyte growth factor supply makes extensive hepatectomy possible in cirrhotic rats. Hepatology 28: 756-769; 1998.

12. Khan, A. A.; Shaik, M. V.; Parveen, N.; Rajendraprasad, A.; Aleem, M. A.; Habeeb, M. A.; Srinivas, G.; Raj, T. A.; Tiwari, S. K.; Kumaresan, K.; Venkateswarlu, J.; Pande, G.; Habibullah, C. M. Human fetal liver-derived stem cell transplantation as supportive modality in the management of end-stage decompensated liver cirrhosis. Cell Transplant. 19:409-418; 2010.

13. Laconi, E.; Pani, P.; Farber, E. The resistance phenotype in the development and treatment of cancer. Lancet Oncol. $1: 235-241 ; 2000$.

14. Laconi, S.; Curreli, F.; Diana, S.; Pasciu, D.; De Filippo, G.; Sarma, D. S. R.; Pani, P.; Laconi, E. Liver regeneration in response to partial hepatectomy in rats treated with retrorsine: A kinetic study. J. Hepatol. 31:1069-1074; 1999.

15. Laconi, S.; Montisci, S.; Doratiotto, S.; Greco, M.; Pasciu, D.; Pillai, S.; Pani, P.; Laconi, E. Liver repopulation by transplanted hepatocytes and risk of hepatocellular carcinoma. Transplantation 82:1319-1323; 2006.

16. Laconi, S.; Pani, P.; Pillai, S.; Pasciu, D.; Sarma, D. S. R.; Laconi, E. A growth constrained environment drives tumor progression in vivo. Proc. Natl. Acad. Sci. USA 98: 7806-7811; 2001

17. Laconi, S.; Pillai, S.; Porcu, P. P.; Shafritz, D. A.; Pani, P.; Laconi, E. Massive liver replacement by transplanted hepatocytes in the absence of exogenous growth stimuli in rats treated with retrorsine. Am. J. Pathol. 158:771$777 ; 2001$.

18. Marongiu, F.; Doratiotto, S.; Montisci, S.; Pani, P.; Laconi, E. Carcinogenesis and liver repopulation: Two sides of the same coin? Am. J. Pathol. 172:857-864; 2008.

19. McLean, E. The toxic actions of pyrrolizidine (senecio) alkaloids. Pharmacol. Rev. 22:429-483; 1970.

20. Moser, M. A.; Kneteman, N. M.; Minuk, G. Y. Research toward safer resection of the cirrhotic liver. HPB Surg. 1: 285-297; 2000

21. Nagasue, N.; Yukaya, H.; Ogawa, Y.; Kohno, H.; Nakamura, T. Human liver regeneration after major hepatic resection. A study of normal liver and livers with chronic hepatitis and cirrhosis. Ann. Surg. 206:30-39; 1987.

22. Seglen, P. O. Preparation of isolated rat liver cells. Methods Cell. Biol. 13:29-83; 1976.

23. Souza, B. S.; Nogueira, R. C.; de Oliveira, S. A.; de Freitas, L. A.; Lyra, L. G.; Ribeiro dos Santos, R.; Lyra, A. C.; Soares, M. B. Current status of stem cell therapy for liver diseases. Cell Transplant. 18:1261-1279; 2009.

24. Thompson, N. L.; Hixson, D. C.; Callanan, H.; Panzica, M.; Flanagan, D.; Faris, R. A.; Hong, W. J.; HartelSchenk, S.; Doyle, D. A Fischer rat substrain deficient in dipeptidyl peptidase IV activity makes normal steady-state RNA levels and an altered protein. Use as a liver-cell transplantation model. Biochem. J. 273:497-502; 1991.

25. Yu, C. H.; Chen, H. L.; Chen, Y. H.; Chang, M. F.; Chien, C. S.; Chang, M. H. Impaired hepatocyte regeneration in acute severe hepatic injury enhances effective repopulation by transplanted hepatocytes. Cell Transplant. 18: 1081-1092; 2009.

26. Zhang, W.; Hanks, A. N.; Boucher, K.; Florell, S. R.; Allen, S. M.; Alexander, A.; Brash, D. E.; Grossman, D. UVB-induced apoptosis drives clonal expansion during skin tumor development. Carcinogenesis 26:249-257; 2005. 
